\title{
Correction to: Individualized home-based exercise and nutrition interventions improve frailty in older adults: a randomized controlled trial
}

Tsung-Jen Hsieh', Shin-Chang Su², Chun-Wei Chen ${ }^{3}$, Yaw-Wen Kang ${ }^{4}$, Ming-Hsia Hü ${ }^{2}$ Li-Lin Hsu', Szu-Yun Wu', Likwang Chen ${ }^{1}$, Hsing-Yi Chang ${ }^{1}$, Shao-Yuan Chuang ${ }^{1}$, Wen-Harn Pan ${ }^{1,6^{*}}$ and Chih-Cheng Hsu ${ }^{1,7,8^{*}}$

\section{Correction to: Int J Behav Nutr Phys Act (2019) 16:119} https://doi.org/10.1186/s12966-019-0855-9

Following publication of the original article [1], the author reported that an abbreviation was incorrect in the original article;

1. In the Appendix the abbreviation 'cal' was incorrect. The correct abbreviation is 'kcal'.

\section{Author details}

'Institute of Population Health Sciences, National Health Research Institutes, 35 Keyan Road, Zhunan, Miaoli County 35053, Taiwan. ${ }^{2}$ School and Graduate, Institute of Physical Therapy, College of Medicine, National Taiwan University, Taipei, Taiwan. ${ }^{3}$ Department of Chest Medicine, Miaoli General Hospital, Miaoli, Taiwan. ${ }^{4}$ Department of Physical Medicine and Rehabilitation, Miaoli General Hospital, Miaoli, Taiwan. ${ }^{5}$ Food Industry Research and Development Institute, Hsinchu, Taiwan. ${ }^{6}$ Institute of Biomedical Sciences, Academia Sinica, 128 Academia Road, Section 2, Nankang, Taipei 11529, Taiwan. 'Department of Health Services Administration, China Medical University, Taichung, Taiwan. ${ }^{8}$ Department of Family Medicine, Min-Sheng General Hospital, Taoyuan, Taiwan.

Published online: 23 December 2019

\section{Reference}

1. Hsieh TJ, et al. Individualized home-based exercise and nutrition interventions improve frailty in older adults: a randomized controlled trial. Int J Behav Nutr Phys Act. 2019;16:119. https://doi.org/10.1186/s12966-0190855-9.

Full list of author information is available at the end of the article

(c) The Author(s). 2019 Open Access This article is distributed under the terms of the Creative Commons Attribution 4.0 International License (http://creativecommons.org/licenses/by/4.0/), which permits unrestricted use, distribution, and reproduction in any medium, provided you give appropriate credit to the original author(s) and the source, provide a link to the Creative Commons license, and indicate if changes were made. The Creative Commons Public Domain Dedication waiver (http://creativecommons.org/publicdomain/zero/1.0/) applies to the data made available in this article, unless otherwise stated. 